\title{
Fatores de risco relacionados a coagulopatias no período gestacional
}

\author{
Risk factors related to coagulopathies in the management period
}

Factores de riesgo relacionados con coagulopatías en el período de gestión

Edilene Oliveira Souza1 ${ }^{1}$ Kátia Helena Campos da Silva ${ }^{1}$, Luiza Bim Vivan ${ }^{1}$, Natália de Oliveira ${ }^{1}$, Gérsika Bitencourt Santos ${ }^{1 *}$.

\begin{abstract}
RESUMO
Objetivo: Identificar os fatores de risco para coagulopatias entre gestantes atendidas em um hospital universitário de Alfenas-MG. Métodos: Foi realizado um estudo documental, transversal, descritivo, de abordagem quantitativa e qualitativa, com análise de prontuários de gestantes assistidas em um hospital universitário do Sul de Minas Gerais. O estudo foi aprovado por Comitê de Ética em Pesquisa humana da Unifenas. Resultados: A média de idade de gestantes foi de vinte e oito anos e três meses, e a maioria realizaram parto cesariano; $1,57 \%$ das gestantes foram acometidas por coagulopatia trombótica anterior à gestação. 2,16\% das mulheres foram submetidas à terapia devido a trombose venosa profunda. Em relação às medicações utilizadas, $73,82 \%$ aderiram ao uso, sendo a maior porcentagem medicamentos de uso rotineiros do período gestacional e apenas $2,17 \%$ fizeram o uso de ácido acetilsalicílico, um antiagregante plaquetário, utilizado antes da gestação. Conclusão: Conclui-se que a maioria das gestantes possuem fatores de risco relacionados a coagulopatias, sendo estes adicionais, relacionados a hábitos de vida, hereditariedade e doenças crônicas, apresentando um número reduzido de usuárias de medicações antitrombóticas.
\end{abstract}

Palavras-chave: Gestação, Coagulopatias, Fatores de risco.

\begin{abstract}
Objective: Identify the risk factors for coagulopathies among pregnant women seen at a university hospital in Alfenas-MG. Methods: A documentary, cross-sectional, descriptive study with a quantitative and qualitative approach was carried out, with analysis of the medical records of pregnant women assisted in a university hospital in the south of Minas Gerais. The study was approved by the human Research Ethics Committee of Unifenas. Results: The average age of pregnant women was twenty-eight years and three months, and the majority underwent cesarean delivery; $1.57 \%$ of pregnant women were affected by thrombotic coagulopathy prior to pregnancy. $2.16 \%$ of women underwent therapy due to deep venous thrombosis. In relation to the medications used, $73.82 \%$ adhered to the use, with the highest percentage being used routinely during pregnancy and only $2.17 \%$ using acetylsalicylic acid, an antiplatelet agent, used before pregnancy. Conclusion: It is concluded that most of pregnant women have risk factors related to coagulopathies, these being additional, related to lifestyle, heredity and chronic diseases, with a reduced number of users of antithrombotic medications.
\end{abstract}

Key words: Pregnancy, Coagulopathies, Risk factors.

\section{RESUMEN}

Objetivo: Identificar los factores de riesgo de coagulopatías en gestantes atendidas en un hospital universitario de Alfenas-MG. Métodos: Se realizó un estudio documental, transversal, descriptivo con abordaje cuantitativo y cualitativo, con análisis de historias clínicas de gestantes atendidas en un hospital universitario del sur de Minas Gerais. El estudio fue aprobado por el Comité de Ética en Investigación em

${ }_{1}^{1}$ Universidade José do Rosário Vellano (UNIFENAS), Alfenas - MG. *E-mail: gersika.santos@unifenas.br

PUBLICADO EM: 2/2021 
humanos de Unifenas. Resultados: La edad promedio de las mujeres embarazadas fue de veintiocho años y tres meses, y la mayoría se sometió a cesárea; El 1,57\% de las mujeres embarazadas sufrió coagulopatía trombótica antes del embarazo. El 2,16\% de las mujeres se sometió a tratamiento debido a una trombosis venosa profunda. En relación a los medicamentos utilizados, el 73,82\% se adhirió al uso, siendo el mayor porcentaje utilizado de forma rutinaria durante el embarazo y solo el 2,17\% utilizando ácido acetilsalicílico, un agente antiplaquetario, utilizado antes del embarazo. Conclusión: Se concluye que la mayoría de las embarazadas presentaba factores de riesgo relacionados con las coagulopatías, siendo estos adicionales, relacionados con el estilo de vida, la herencia y las enfermedades crónicas, con un número reducido de usuarios de medicamentos antitrombóticos.

Palabras clave: Embarazo, Coagulopatías, Factores de riesgo.

\section{INTRODUÇÃO}

A embolia é entendida como a deslocação de porções de trombos, gordura, gases ou corpos estranhos pela corrente sanguínea a locais diferentes de seu princípio e a sua maioria equivale ao tromboembolismo, sendo esta a locomoção para a circulação pulmonar ou sistêmica (GOUVEIA M, et al., 2016). Segundo Rybstein MD e DeSancho MT (2019), deve- se ficar atento para os fatores de risco que contribuem para o tromboembolismo na gestação, as mulheres com histórico pessoal ou familiar de TEV, presença de anticorpos antifosfolipídios, tabagismo, obesidade, idade materna > 35 anos, multiparidade, lúpus eritematoso sistêmico, pré eclâmpsia. Além disso, observa-se que durante a gestação ocorre a hipercoagulabilidade fisiológica, que somado a questões variadas predispõem e aumentam as chances de tromboembolismo.

Um fator determinante para esse acontecimento é a coagulação, pelo qual surgem vários componentes na superfície de uma lesão, que atingem a agregação plaquetária e formam o tampão plaquetário. No período gestacional e no puerpério tem-se a formação da Tríade de Virchow, caracterizado pela estase sanguínea, presente principalmente nos membros inferiores (MMII), que evolui para a ocorrência de alterações das paredes dos vasos devido à pressão exercida pelo útero gravídico, principalmente nos pélvicos, e mudança na cascata de coagulação (COSTA FLP, 2007).

Segundo o Brasil (2018), considerando os diversos tipos de coagulopatias hereditárias, no Brasil a minoria $32,59 \%$ são pacientes do sexo feminino, nas coagulopatias hereditárias raras $50,66 \%$ são do sexo feminino e nas outras coagulopatias e demais transtornos hemorrágicos $63,98 \%$ são do sexo feminino. Destaca-se que a maior prevalência ocorre na faixa etária dos 20-29 anos de idade. Se comparar com os dados de 2012 ocorreu um aumento de casos. Um estudo de Yang YY, et al. (2018), realizado com as pacientes internadas em estado crítico da maternidade no primeiro hospital afiliado da Anhui Medical University de janeiro de 2012 a setembro 2015, indicou que as principais causas de morbidade materna grave foram transfusão maciça de sangue, trombocitopenia, disfunção de coagulação, quadro clínico de choque e remoção do útero induzida por infecção ou sangramento do útero. Percebe-se que os distúrbios que envolvem coagulopatias estão entre as principais causas e devem ser monitoradas para a redução da morbimortalidade materna.

Nota-se que o diagnóstico do tromboembolismo durante a gestação depende de exames de imagem objetivos e utiliza-se os testes auxiliares como regras de predição clínica que são importantes para um tratamento eficaz (CHAN WS, 2017). Segundo Andersen AS, et al. (2015), é de suma importância o diagnóstico precoce e o início da profilaxia em gestações de risco para evitar a progressão da patologia e embolia pulmonar.

Este artigo tem como objetivo identificar os fatores de risco para coagulopatias em gestantes internadas em um hospital universitário, identificar patologias diagnosticadas que aumentam o risco de coagulopatias, relatar os principais exames laboratoriais realizados pelas gestantes e relatar os principais medicamentos utilizados pelas pacientes. 


\section{MÉTODOS}

Estudo documental, retrospectivo, de abordagem quantitativa e qualitativa, com análise de prontuários de gestantes assistidas em um hospital universitário da cidade de Alfenas - Minas Gerais. A coleta de dados aconteceu em 2019 referente aos prontuários dos seis primeiros meses do mesmo ano. A coleta de dados foi realizada através da análise de prontuários de gestantes em regime de internação. Foram extraídas informações de cento e noventa prontuários, referente aos aspectos clínicos, pessoais e conduta terapêutica, englobando clientes de toda faixa etária e idade gestacional. O trabalho foi aprovado pelo comitê de ética em pesquisa humana da Unifenas sob parecer no 3.288.549. Os dados foram tabulados em um banco de dados elaborado pelos pesquisadores e organizados no Microsoft Excel 2010.

\section{RESULTADOS E DISCUSSÃO}

A média de idade das gestantes estudadas foi de vinte e oito anos, sendo a maior porcentagem compreendida entre 19 a 29 anos (54,98\%) (Gráfico 1). Neste estudo, a faixa etária mais prevalente das gestantes está dentro do preconizado pelo MS. Segundo Oliveira ALML e Marques MA (2016), as mulheres com mais de 35 anos tem aumento de risco de duas vezes para eventos tromboembólicos, principalmente as mulheres em fase puerperal. No presente estudo a maior porcentagem das gestantes internadas foi entre 19 e 29 anos, faixa etária inferior a que é considerada de risco. O MS recomenda que a faixa de idade considerada ideal para engravidar é dos 19 aos 32 anos, tendo em vista que a partir dos 35 anos, no caso de primigestação, a gravidez é considerada de alto risco (MEIRELES JFF, et al. 2016).

Gráfico 1 - Percentual de faixa etária das gestantes estudadas.

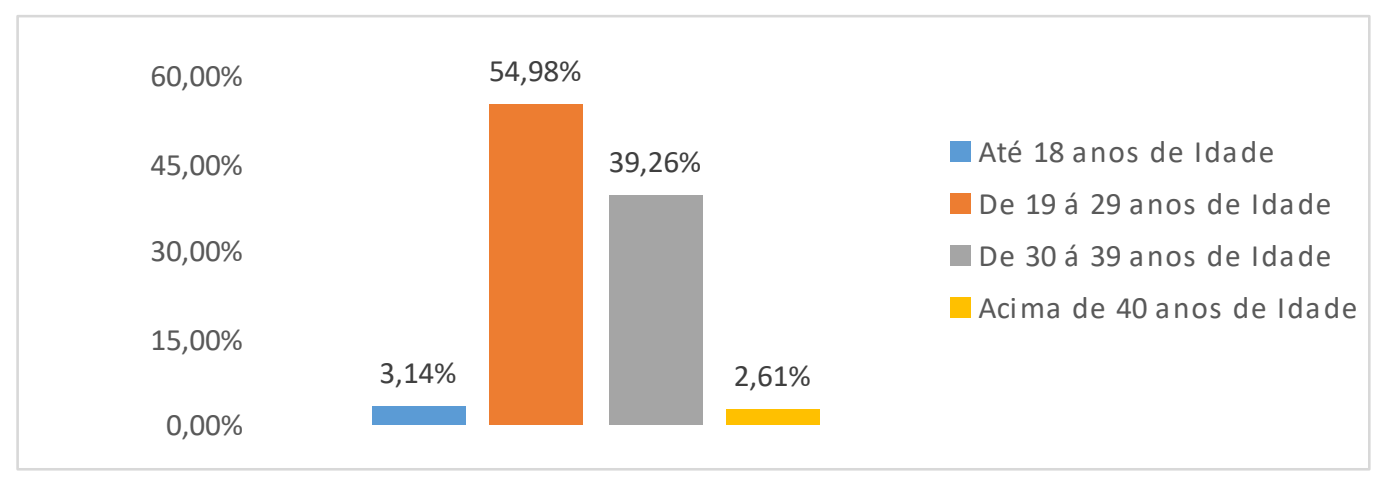

Fonte: Souza EO, et al., 2021.

A média da idade gestacional foi de trinta e seis semanas; e o percentual de $91,10 \%$ compreende-se o terceiro trimestre (Gráfico 2). No estudo predominou-se a gestação multípara com $61,25 \%$, seguida da primípara com $38,74 \%$. Os índices de parto expuseram um maior percentual em cesariana $(55,49 \%)$, em sequência o parto vaginal $(38,21 \%)$, seguidos por abortos $(3,66 \%)$ e curetagem $(2,61 \%)$.

Gráfico 2 - Média da Idade Gestacional das pacientes estudadas.

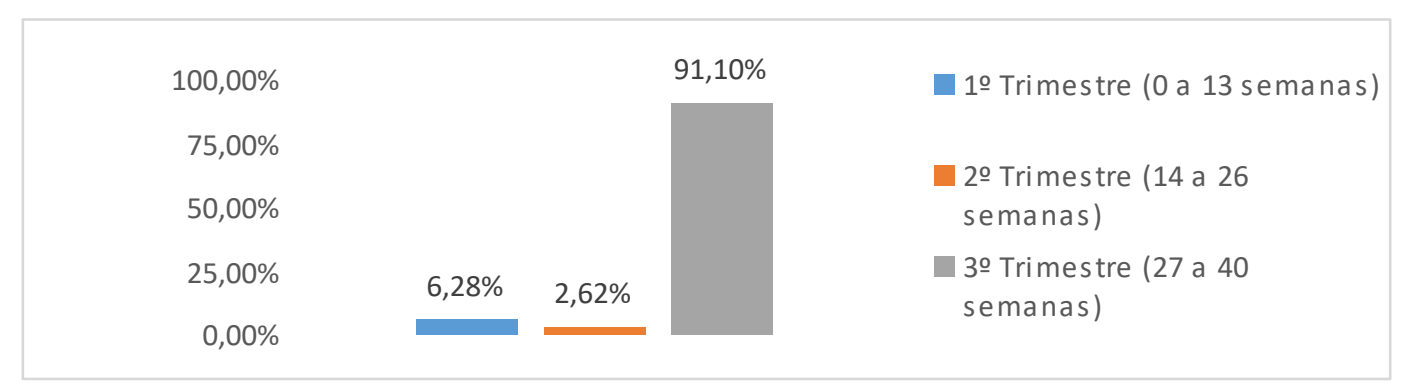

Fonte: Souza EO, et al., 2021. 
Em relação à idade gestacional houve predominância entre 27 e 40 semanas, enquadradas no terceiro trimestre, adequado por ser um período notável de crescimento e desenvolvimento intrauterino (MEIRELES JFF, et al. 2016).

Apesar de que estudos de Greer IA (2012), afirmam que a mulher estar grávida aumenta o risco de tromboembolismo venoso de cinco a dez vezes, podendo chegar a 35 vezes no puerpério, quando compara com as mulheres não gestantes da mesma idade. Nesse estudo, não teve um aumento significativo, e pode ser devido a idade materna da maioria ser menor que 35 anos. Ressalta-se que a prevenção na gestação e no puerpério é a melhor estratégia para prevenir e aliado a isso é o diagnóstico precoce das pacientes.

Segundo o Royal College of Obstetricians and Gynaecologists (RCOG) (2015), as pacientes assintomáticas que apresentam deficiência de antitrombina, proteína $C$ ou $S$, mutação do gene da protrombina ou anticorpos antifosfolípideos devem ser indicadas para profilaxia pré natal. Lembra-se que todas as mulheres submetidas a cesariana devem realizar profilaxia durante 10 dias, exceto as cesarianas eletivas que só aceitam profilaxia na presença de fatores de risco adicionais.

Os resultados encontrados evidenciam um percentual maior de parto cesáreo em relação ao parto vaginal. Segundo Campana HCR e Pelloso SM (2007), um estudo realizado sobre o perfil epidêmico detectou elevada proporção da prática da cesárea em mulheres com número maior de cesarianas anteriores, sendo os fatores associados à indicação da via de resolução do parto algumas condições como a socioeconômica, a indicação médica, a opção da parturiente ou do obstetra, as condições institucionais e os antecedentes de cesárea. O risco de trombose venosa profunda é sugerido com um maior índice em mulheres submetidas ao parto via cesárea, consoante com o Parecer Técnico no 01/2017 da Comissão Permanente de Farmácia e Terapêutica de Mato Grosso (CPFT-MT).

Em relação ao desenvolvimento da trombose venosa profunda (TVP), 1,58\% foram acometidas no período anterior à gestação e $98,42 \%$ não desenvolveram TVP antes, durante ou após a gravidez. De acordo com Machado CGE, et al. (2017), a incidência de risco absoluto de trombose é de apenas $0,1 \%$, o que explica o baixo índice de gestante que apresentaram trombose venosa profunda e nos relatos registrados desse distúrbio de coagulação, ocorreram anteriores ao período gestacional.

De acordo com o estudo de Kalil JA ,et al. (2008), a trombose venosa profunda (TVP) ocorreu com predomínio no terceiro trimestre da gravidez. No entanto, difere do estudo presente, em que as gestantes que apresentaram TVP foi no período anterior a gestação.

O desempenho dos exames para as gestantes apresentam dificuldades, devido ao risco associado a exposição do feto a radiação e preocupações relacionadas á administração de agente do contraste (CHAN WS, et al., 2010). Como também, o biomarcador dímero $D$ possui limitações durante a gestação, pois aumenta fisiologicamente, principalmente no terceiro trimestre (GARCIA IG, et al., 2018). Observa-se que as pacientes do estudo não foram submetidas a esse exame laboratorial.

Dentre as mulheres que foram submetidas a terapia devido a TVP, algumas apresentavam com préeclampsia ou já tiveram abortos repetidos. O documento do Ministério da Saúde, Conitec (2017), mostra que o uso da enoxaparina está associado a uma chance 15,5 vezes maior de gestações bem-sucedidas (número de nascidos vivos) com valores estatisticamente significantes, quando comparado com gestantes, nas situações descritas acima, sem o uso do anticoagulante.

Dos fatores de risco apresentados pelas gestantes internadas, os percentuais maiores caracterizaram o diabetes mellitus (14,66\%), tabagismo (6,80\%), hipotireoidismo (4,92\%), etilismo (2,10\%), Ruptura Prematura de Membranas Ovulares (RPMO) (1,97\%) e hipertensão arterial gestacional (1,58\%). Ao analisar a presença de doenças crônicas, $86,91 \%$ não apresentaram nenhuma doença crônica, $4,71 \%$ apresentava diagnóstico de diabetes mellitus, o mesmo percentual para hipertensão arterial sistêmica (HAS), 2,09\% têm doenças pulmonares, $1,04 \%$ são cardiopatas e 0,52\% são soropositivas para o Vírus da Imunodeficiência Humana (HIV).

O diabetes mellitus predispõe o paciente a eventos trombóticos, visto que as placas ateroscleróticas são mais vulneravéis, apresentam desregulação tanto no receptor quanto nos níveis de transdução de sinal 
intracelular, como também ocorre aumento da quantidade de lipídios extracelular , inflamação e estado prótrombótico. Além disso, a hiperglicemia aumenta a produção de espécies reativas de oxigênio que tem como consequência a contribuição para disfunção endotelial e possui efeitos diretos na transcrição gênica dos fatores de coagulação. Observa-se também que os pacientes com diabetes possuem um aumento da geração de trombina e fibrina e retardo da lise do coágulo (PATTI G, 2019). Por isso, é necessário um cuidado maior com as gestantes que possuem essa patologia, visto que foi o fator de risco predominante no estudo e se não estiver controlada pode ter sérias consequências, tanto para a paciente como para o feto.

Entre os fatores de risco das gestantes do estudo, o tabagismo está relacionado com o hábito de vida e teve uma porcentagem mais alta que as doenças metabólicas como hipotireoidismo, por isso a importância de informar a paciente dos riscos. Segundo Charlo PB, et al. (2020), o cigarro gera um dano na parede vascular, com elevação dos níveis de fibrinogênio plasmático, por conseguinte ativa a cascata de coagulação, e a nicotina induz o estado pró-trombótico por ativação plaquetária.

Tomando por referência o Parecer Técnico no 01/2017 da Comissão Permanente de Farmácia e Terapêutica de Mato Grosso (CPFT-MT), ainda existem outros fatores de risco adicionais que podem estar presentes na gestação, aumentando a chance de fenômenos tromboembólicos, dentre eles, história prévia de tromboembolismo, história pessoal ou familiar de trombofilia, idade superior a 35 anos e obesidade (IMC superior a $\left.29 \mathrm{Kg} / \mathrm{m}^{2}\right)$.

Segundo Machado CGE, et al. (2017), outros fatores podem aumentar o risco de tromboembolismo venoso, tais como a idade maior que 35 anos, cirurgia recente, paridade aumentada, obesidade, tabagismo, imobilização, história pessoal anterior de tromboembolismo venoso (TEV) ou histórias de TEV de parentes de primeiro grau. Em relação ao histórico familiar, os maiores índices identificados evidenciaram a hipertensão arterial sistêmica (25,33\%), diabetes mellitus (15,84\%), cardiopatias $(6,34 \%)$, câncer $(2,09 \%)$ e doenças pulmonares $(1,35 \%)$.

A hipertensão arterial é considerada uma das mais importantes complicações do ciclo gravídicopuerperal, com incidência em $6 \%$ a $30 \%$ das gestantes, e resulta em alto risco de morbidade e mortalidade materna e perinatal (ASSIS TR, et al., 2008).

A gestação é um estado hiperinsulinêmico caracterizado por uma diminuição da sensibilidade à insulina, parcialmente explicada pela presença de hormônios diabetogênicos, tais como a progesterona, o cortisol, a prolactina e o hormônio lactogênico placentário. Os níveis glicêmicos de jejum tendem a ser mais baixo na gestante, contudo, os valores pós-prandiais são mais altos, sobretudo naquelas em que não há aumento adequado da liberação de insulina (MAGANHA CA et al., 2003).

Segundo Meneguin S e Xavier CL (2013), a associação entre doença cardíaca e gravidez é reconhecidamente fator de risco obstétrico e fetal em potencial durante o ciclo gravídico-puerperal, sendo as cardiopatias consideradas as principais causas indireta de mortalidade materna em nível mundial.

Em relação ao câncer, Kettelhut JC e Modena MAB (2008), relatam que devido às alterações fisiológicas próprias do ciclo gravídico-puerperal, as gestantes possuem maior risco de apresentar o câncer de mama em estágio avançado, pois nódulos pequenos são difíceis de ser diagnosticados. Citando as doenças pulmonares, as doenças cardiorrespiratórias são causas importantes de morbidade e mortalidade em gestantes, pois, durante a gestação o aumento do volume abdominal reduz a eficiência dos movimentos respiratórios diminuindo a expansibilidade pulmonar, levando a modificações importantes no sistema (CHICAYBAN LM e DIAS S, 2010).

Em relação à farmacoterapia, $26,17 \%$ das gestantes não utilizavam qualquer tipo de medicamento. Das gestantes usuárias de algum fármaco, 73,82\% realizaram uso de sulfato ferroso (17,06\%), polivitamínicos $(16,13 \%)$, levotiroxina sódica $(8,68 \%)$, metildopa $(4,96 \%)$, ácido fólico $(3,73 \%)$ e insulina $(2,48 \%)$ (Gráfico 3). 
Gráfico 3 - Medicamentos utilizados pelas pacientes durante a gestação.

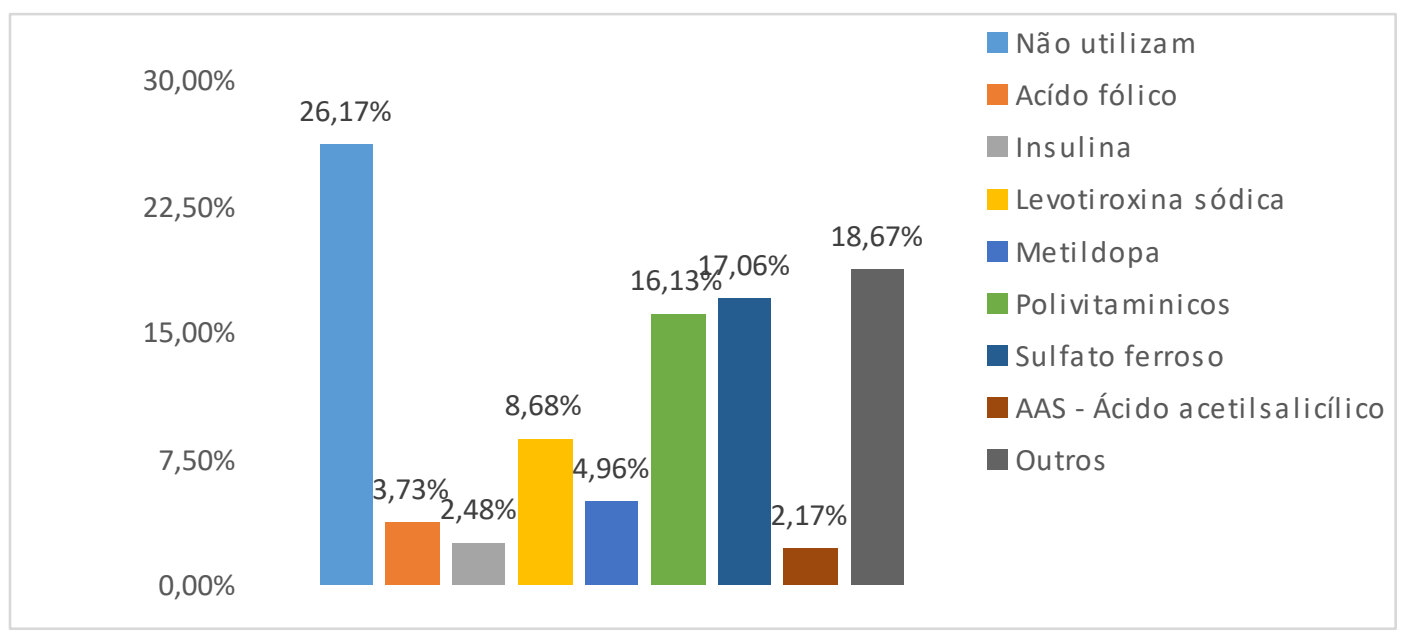

Fonte: Souza EO, et al., 2021.

Houveram utilizações por gestantes em predominância de sulfato ferroso e polivitamínicos, evidência também explicitada em outros estudos (NASCIMENTO AM, et al., 2016). Embora tenha sido identificado em um menor índice, o uso de levotiroxina sódica foi considerável, uma vez que possui correlação com o distúrbio hormonal evidenciado consideravelmente no resultado da pesquisa. Não houveram citações dessa última medicação em outros artigos nas pesquisas levantadas.

Dos medicamentos encontrados em uso, apenas o ácido acetilsalicílico (AAS), um antiagregante plaquetário se encaixa como adequado na terapia para trombose venosa profunda, pois é o único medicamento a ser utilizado pela gestante em âmbito ambulatorial, sendo realizado na maioria das vezes uma pausa perto ao momento do parto (MINISTÉRIO DA SAÚDE, 2017). Segundo James AH (2009); os agentes preferidos para anticoagulação na gestação são os compostos de heparina, pois tanto a heparina não fracionada e a fracionada são consideradas seguras e não atravessam a placenta, ou seja não prejudica o feto.

A aspirina durante á gravidez pode ter seu efeito reduzido, pois durante a gestação ocorre um aumento da renovação plaquetária, com plaquetas imaturas propensas a se ativar e agregar mais rapidamente (NAVARATNAM K, et al., 2018). Diante disso, é necessário acompanhar as gestantes que fazem uso deste medicamento, no estudo é a minoria , somente $2,17 \%$.

Dos exames prévios, $85,34 \%$ realizaram hemograma, 0,52\% a dosagem de protrombina e $14,13 \%$ não realizaram qualquer tipo de exame laboratorial. Para as mulheres que se submeteram a terapia com antitrombóticos, os exames realizados e os maiores resultados foram para Tempo de Tromboplastina Parcialmente Ativada (TTPA) $(3,66 \%)$ e contagem de plaquetas $(0,52 \%)$.

Os testes laboratoriais auxiliam no diagnóstico da disfunção plaquetária, como também são utilizados para monitoração de drogas antiplaquetárias, terapia pró-hemostáticas e prevenção de trombose ou sangramento. O TTPA é o teste de triagem que avalia os fatores das vias intrínseca e comum da coagulação. No caso de disfunções plaquetárias por agregação plaquetária repete-se sempre o exame para confirmação do diagnóstico. Outro fator importante é algumas substâncias ingeridas que podem interferir na função plaquetária, tanto medicamentos como alimentos e complementos (BRASIL, 2016).

Diante do aumento das coagulopatias no sexo feminino, é necessário um acompanhamento pré natal para prevenir e tratar as coagulopatias nas pacientes gestantes. A garantia desses cuidados é condição fundamental para uma gestação segura, tanto para a mãe como para o feto. Como afirma Friedman AM e Ananth CV (2016), a gravidez já é um fator de risco para tromboembolismo venoso, os fatores de risco tanto de hábitos de vida como comorbidades médicas estão cada vez mais presentes e prejudicam mais o quadro clínico da paciente. Porém, com acompanhamento é possível a prevenção e em alguns casos é utilizado a tromboprofilaxia. 


\section{CONCLUSÃO}

Uma porcentagem considerável das gestantes estudadas apresentou fatores de risco relacionados à coagulopatias relacionados a hábitos de vida, hereditariedade e doenças crônicas, visto que o percentual maior nas pacientes internadas eram as que apresentavam diabetes mellitus, seguido por tabagismo. Porém, observou-se que no presente estudo teve um número reduzido de casos de TVP e de gestantes usuárias de medicações antitrombóticas. Dentre os fármacos mais utilizados pelas mulheres destacam-se os suplementos como ácido fólico, sulfato ferroso e polivitamínicos, comumente utilizados no período gestacional, hormonal (levotiroxina sódica), anti-hipertensivo (metildopa) e antidiabético (insulina), todos medicamentos considerados seguros para utilização durante a gestação.

\section{REFERÊNCIAS}

1. ANDERSEN AS, et al. Pregnancy predispose to higher incidence of venous thromboembolism. Ugeskrift for laeger, $2015 ; 177$.

2. ASSIS TR, et al. Estudo dos principais fatores de risco maternos nas síndromes hipertensivas da gestação. Arquivos Brasileiros de Cardiologia, 2008; 91.

3. BRASIL. Manual do Ministério da Saúde, 2016.

4. BRASIL. Ministério da Saúde. Perfil das coagulopatias hereditárias no Brasil 2016. Brasília, DF, 2018.

5. CAMPANA HCR, PELLOSO SM. Levantamento dos partos cesárea realizados em um hospital universitário. Rev. Eletr. Enf, 2007; 9(1):51-63.

6. COSTA FLP, et al. Trombose venosa profunda na gestação: conhecimento e pratica profissional. Revista Enfermeira Global. America Latina, 2007.

7. CHAN WS. Diagnosis of venous thromboembolism in pregnancy. Elsevier, 2017.

8. CHAN WS, et al. Anatomic distribution of deep vein thrombosis in pregnancy. CMAJ, 2010.

9. CHARLO PB, et al.Relação entre trombose venosa profunda e seus fatores de risco na população feminina. Glob Acad Nurs, 2020.

10. CHICAYBAN LM, DIAS S. Análise da função pulmonar em gestantes e não-gestantes.Perspectivas Online,2010; 4(15).

11. FRIEDMAN AM, ANANTH CV. Obstetrical venous thromboembolism: Epidemiology and strategies for prophylaxis. Seminars in Perinatology, 2016; 40.

12. GARCIA IG, et al. D-dimer during pregnancy: establishing trimester-specific reference intervals. Scand J Clin Lab Invest, 2018.

13. GOUVEIA M, et al. Embolia Pulmonar em Portugal: Epidemiologia e Mortalidade Intra-Hospitalar, 2016.

14. GREER IA. Thrombosis in pregnancy: updates in diagnosis and management. Hematology Am Soc Hematol Educ Program, 2012.

15. JAMES AH. Venous Thromboembolism in Pregnancy. Arterioscler Thromb Vasc Biol, 2009.

16. KALIL JA, et al. Investigação da trombose venosa na gravidez. Journal Vascular Brasileiro. Porto Alegre,2008; 7(1).

17. KETTELHUT JC, MODENA MAB. Câncer de mama e gestação. Revista da Faculdade de Ciências Médicas de Sorocaba, 2008; 10(4).

18. MACHADO CGE, et al. Sistema de Gestão de Qualidade. Protocolo Clínico: Tromboembolismo na Gestação, 2017.

19. MAGANHA CA, et al. Tratamento do diabetes melito gestacional. Rev. Assoc. Med. Bras, 2003; 49(3):330-334.

20. MENEGUIN S, XAVIER CL. Qualidade de vida em gestantes com cardiopatia. Rebita Texto e Contexto Enfermagem, 2013; 22(3).

21. MEIRELES JFF, et al. Satisfação corporal, idade gestacional e estado nutricional em gestantes. ABCS Health Sciences, 2016.

22. MINISTÉRIO DA SAÚDE. CONITEC, Comissão Nacional de Incorporação de Tecnologias do Sus. Enoxaparina para gestantes com trombofilia, 2017.

23. NAVARATNAM K, et al. Aspirin non-responsiveness in pregnant women at high-risk of pre-eclampsia. Eur J Obstet Gynecol Reprod Biol, 2018.

24. NASCIMENTO AM, et al. Avaliação do uso de medicamentos por gestantes em Unidades Básicas de Saúde de Rondonópolis, Mato Grosso. Revista Eletrônica Gestão \& Saúde, 2016.

25. OLIVEIRA ALML, MARQUES MA. Profilaxia de tromboembolismo venoso na gestação. Jornal Vascular Brasileiro. Porto Alegre, 2016; 15(4).

26. PATTI G, et al. Prevention of atherothrombotic events in patients with diabetes mellitus: from antithrombotic therapies to new-generation glucose-lowering drugs. Nat Rev Cardiol, 2019.

27. RCOG. Reducing the Risk of Venous Thromboembolism during Pregnancy and the Puerperium. Royal College of Obstetricians and Gynaecologists (Green-top Guideline), 2015; (37).

28. YBSTEIN MD, DESANCHO MT. Risk Factors for and Clinical Management of Venous Thromboembolism During Pregnancy. Clinical Advances in Hematology \& Oncology, 2019; 17.

29. YANG YY, et al. A retrospective cohort study of risk factors and pregnancy outcomes in 14,014 Chinese pregnant women. Medicine, 2018; 97. 